\title{
Clinical application of targeted next-generation sequencing for colorectal cancer patients: a multicentric Belgian experience
}

\author{
Nicky D'Haene ${ }^{1}$, Quitterie Fontanges ${ }^{1}$, Nancy De Nève ${ }^{1}$, Oriane Blanchard ${ }^{1}$, Barbara \\ Melendez ${ }^{1}$, Monique Delos ${ }^{2}$, Marie-Françoise Dehou ${ }^{3}$, Calliope Maris ${ }^{1,4}$, Nathalie \\ Nagy $^{5}$, Emmanuel Rousseau ${ }^{6}$, Josse Vandenhove ${ }^{7}$, André Gilles ${ }^{8}$, Carine De Prez ${ }^{9}$, \\ Laurine Verset ${ }^{1,10}$, Marie-Paule Van Craynest ${ }^{11}$, Pieter Demetter ${ }^{1}$, Jean-Luc Van \\ Laethem $^{12}$, Isabelle Salmon ${ }^{1}$ and Marie Le Mercier ${ }^{1}$ \\ ${ }^{1}$ Department of Pathology, Erasme Hospital, Université Libre de Bruxelles, Brussels, Belgium \\ ${ }^{2}$ Department of Pathology, CHU UCL Namur, Yvoir, Belgium \\ ${ }^{3}$ CMP Pathology Laboratory, Brussels, Belgium \\ ${ }^{4}$ Department of Pathology, Braine I'Alleud Waterloo Hospital, Braine I'Alleud, Belgium \\ ${ }^{5}$ Department of Pathology, Charleroi University Hospital, Charleroi, Belgium \\ ${ }^{6}$ Department of Pathology, Mouscron Hospital, Mouscron, Belgium \\ ${ }^{7}$ Department of Pathology, Sint Maria Hospital, Halle, Belgium \\ ${ }^{8}$ Department of Pathology, EPICURA Hospital, Frameries, Belgium \\ ${ }^{9}$ Department of Pathology, Brugmann University Hospital, Brussels, Belgium \\ ${ }^{10}$ CurePath, Jumet, Belgium \\ ${ }^{11}$ New LabPatho, Braine I'Alleud, Belgium \\ ${ }^{12}$ Department of Oncology, Erasme Hospital, Université Libre de Bruxelles, Brussels, Belgium \\ Correspondence to: Nicky D'Haene, email: nicky.d.haene@erasme.ulb.ac.be \\ Keywords: colorectal cancer; next-generation sequencing \\ Abbreviations: CRC: Colorectal Cancer; NGS: Next-generation sequencing; FFPE: formalin-fixed paraffin-embedded; TAT: \\ turnaround time \\ Received: December 12, $2017 \quad$ Accepted: March 17, $2018 \quad$ Published: April 17, 2018 \\ Copyright: D'Haene et al. This is an open-access article distributed under the terms of the Creative Commons Attribution License \\ 3.0 (CC BY 3.0), which permits unrestricted use, distribution, and reproduction in any medium, provided the original author and \\ source are credited.
}

\section{ABSTRACT}

International guidelines made RAS (KRAS and NRAS) status a prerequisite for the use of anti-EGFR agents for metastatic colorectal cancer (CRC) patients. Daily, new data emerges on the theranostic and prognostic role of molecular biomarkers; this is a strong incentive for a validated, sensitive, and broadly available molecular screening test. Next-generation sequencing (NGS) has begun to supplant other technologies for genomic profiling. We report here our 2 years of clinical practice using NGS results to guide therapeutic decisions.

The Ion Torrent AmpliSeq colon/lung cancer panel, which allows mutation detection in 22 cancer-related genes, was prospectively used in clinical practice (BELAC ISO 15189 accredited method). The DNA of 741 formalin-fixed paraffinembedded CRC tissues, including primary tumors and metastasis, was obtained from 14 different Belgian institutions and subjected to targeted NGS.

Of the tumors tested, $98 \%$ (727) were successfully sequenced and $89 \%(650)$ harbored at least one mutation. KRAS, BRAF and NRAS mutations were found in 335 $(46 \%), 78(11 \%)$ and $32(4 \%)$ samples, respectively. These mutation frequencies were consistent with those reported in public databases. Moreover, mutations and amplifications in potentially actionable genes were identified in 464 samples (64\%), 


\begin{abstract}
including mutations in PIK3CA (14\%), ERBB2 (0.4\%), AKT1 $(0.6 \%)$, and MAP2K1 $(0.1 \%)$, as well as amplifications of ERBB2 $(0.3 \%)$ and EGFR $(0.3 \%)$. The median turnaround time between reception of the sample in the laboratory and report release was 8 calendar days.

Overall, the AmpliSeq colon/lung cancer panel was successfully applied in daily practice and provided reliable clinically relevant information for CRC patients.
\end{abstract}

\section{INTRODUCTION}

Colorectal Cancer (CRC) is the second most frequent cancer in Europe, irrespective of gender, and still yields a high mortality rate, accounting for $12 \%$ of cancer deaths [1]. Despite a broad screening program, $25 \%$ of the patients are metastatic at initial diagnosis. Moreover, one out of two patients will develop metastasis [1].

Current therapeutical guidelines for stage IV patients include a combination of cytotoxic and biological targeted agents [2]. The approved agents are monoclonal antibodies targeting epidermal growth factor receptor (EGFR): cetuximab and panitumumab. Until recently, indications for standard-of-care molecular testing in colorectal carcinomas included testing for KRAS mutational status as a predictor of response to anti-EGFR agents [3]. Now, American and European guidelines clearly emphasize expanded RAS (KRAS and NRAS) status as a mandatory precondition for use of anti-EGFR therapy [2,4]. Indeed, not only is the benefit of anti-EGFR therapy confined to RAS wild type (wt) tumors, but treatment with anti-EGFR antibodies may even harm patients with a RAS mutation. BRAF mutation is a strong negative prognostic biomarker and evidence is accumulating that patients with a BRAF mutant tumor do not benefit from anti-EGFR therapy $[2,5]$.

Promising targeted therapy and personalized medicine are making molecular profiling of tumors a priority. International efforts to catalogue mutations for multiple forms of cancer, coupled with the successes of targeted agents in patients with molecularly defined tumors, have generated enthusiasm for incorporating genomic profiling into clinical cancer practice. Daily, new data emerges on the theranostic and prognostic role of molecular biomarkers. This is a strong incentive for a validated, sensitive and broadly available molecular screening test in order to implement and improve multimodal therapy strategy and clinical trials.

We have recently validated and accredited (BELAC ISO 15189) the use of the Ion Ampliseq ${ }^{\mathrm{TM}}$ Colon and Lung cancer panel on the Ion Torrent Personal Genome Machine (PGM - Life Technologies) for screening lung and colorectal cancers. This NGS panel is a multiplex PCRbased library preparation method by which 90 amplicons that encompasses 1825 mutational hotspots of 22 genes related to colon and lung cancer are selectively amplified and sequenced.

In the present study, we evaluated the use of this panel on 741 samples from different institutions that have been tested in the context of daily practice since the accreditation of the technique.

\section{RESULTS}

\section{Clinical series}

A total of 741 FFPE samples from patients with colorectal cancer were received from 14 different institutions for NGS testing from November 2013 to December 2015, each contributing from 1 to 153 samples (Figure 1). For two cases $(0.3 \%)$, sequencing could not be performed because of insufficient tumor tissue left. The type of the sample was recorded for 708 patients; the series included 390 surgical resections (55\%), 311 biopsies (44\%) and 7 cell blocks (1\%). The site of the sample was recorded for 696 patients; the primary tumor was tested for 584 patients $(84 \%)$ and metastasis for 112 patients $(16 \%)$ (Table 1). According to international guidelines, either primary or metastatic specimens may be used depending on the particular case requirements $[6,7]$.

\section{Sequencing performances}

Sequencing performance was assessed from the number and distribution of sequencing reads across the targeted regions. Among the 741 sequenced cases, 727 (98\%) were informative (Table 1). Average base coverage of all samples was $1,493 \times$. Six cases with a coverage between 300 and $500 \times$ were also reported with a comment about the suboptimal coverage and the few exceptional cases (19) with average base coverage $<500 x$ and with relevant mutations that passed the quality criteria (Material and Methods) were identified. These mutations were reported because of their high clinical impact.

\section{Turnaround time}

It is important that clinically relevant targets are reported within a clinically useful timeframe. International guidelines recommend that RAS results should be available within 7-10 working days of receiving the specimen in the testing laboratory [6-9]. We tracked the turnaround time (TAT) by retrieving status data from the laboratory information system. The median TAT between reception of the sample in the laboratory and report release was 8 calendar days. For $76 \%$ of the cases, the report was released within 10 calendar days after receiving the samples (Figure 2). Only 17 cases (2\%) had a TAT above 
10 working days, which was due to samples requiring reanalysis, interpretation difficulties and organizational delays.

\section{Overview of identified variants}

The number of mutations per tumor ranged from 0 to 5 (mean 1.6). In the majority of the cases (534/727; $73 \%$ ) only 1 or 2 mutations were detected. In 77 cases $(11 \%)$ no mutations were found in any of the analyzed regions.

The most frequent mutations were found in TP53 (62\%) and KRAS (46\%) (Table 2 and Figure 3). Of successfully sequenced cases, 563 potentially actionable mutations were identified in 464 patients $(64 \%)$, including 343 KRAS mutations, 32 NRAS mutations, 78 BRAF mutations, 101 PIK3CA mutations, 4 AKT1 mutations, 3 ERBB2 mutations, 1 EGFR mutation, and 1 MAP2K1 mutation. The frequencies of these variants detected by NGS were consistent with frequencies reported in public databases (www.cbioportal.org; http://cancer.sanger.ac.uk/ cosmic).

The detection of mutations by NGS showed high sensitivity. Eighty-three, 89 and $91 \%$ of the cases with $<10 \%, 10-50 \%$, and $>50 \%$ tumor cell content, respectively, showed at least one mutation. Furthermore, around $50 \%$ of cases showed KRAS and/or NRAS mutations for each category of tumor cell content $(27 / 54$ in cases with $<10 \%$ tumor cell content; $278 / 553$ in those with $10-50 \%$ tumor cells; or $59 / 117$ in cases with $>50 \%$ tumor cells).

\section{Gene amplification detection}

Using coverage analysis, potential EGFR or ERBB2 amplifications were suggested for 2 and 2 patients, respectively. Indeed, in 2 cases the mean coverage for the 90 amplicons was of $1,458 \times$ and $1,413 \times$, whereas the mean coverage of the 5 EGFR amplicons was of $6,086 \times$ and $12,508 \times$, respectively, suggesting the presence of an EGFR gene amplification (Figure 4). For the 2 other cases, the mean coverage for the 90 amplicons was of $1,366 \times$ and $1,329 \times$, whereas the mean coverage of the 3 ERBB2 amplicons was of $6,432 \times$ and $15,019 \times$, respectively (Figure 4). For these 4 cases, the suggested amplification was confirmed by ISH (Supplementary Figure 1).

\section{DISCUSSION}

A major advantage of NGS over traditional mutation detection methods is its ability to screen multiple mutations in multiple genes simultaneously without the need to perform several sequential tests. Several studies have already validated the use of NGS and its superiority in terms of sensitivity and speed compared to traditional methods [10-13]. In our own experience, for tests including more than two to three different hotspots, NGS

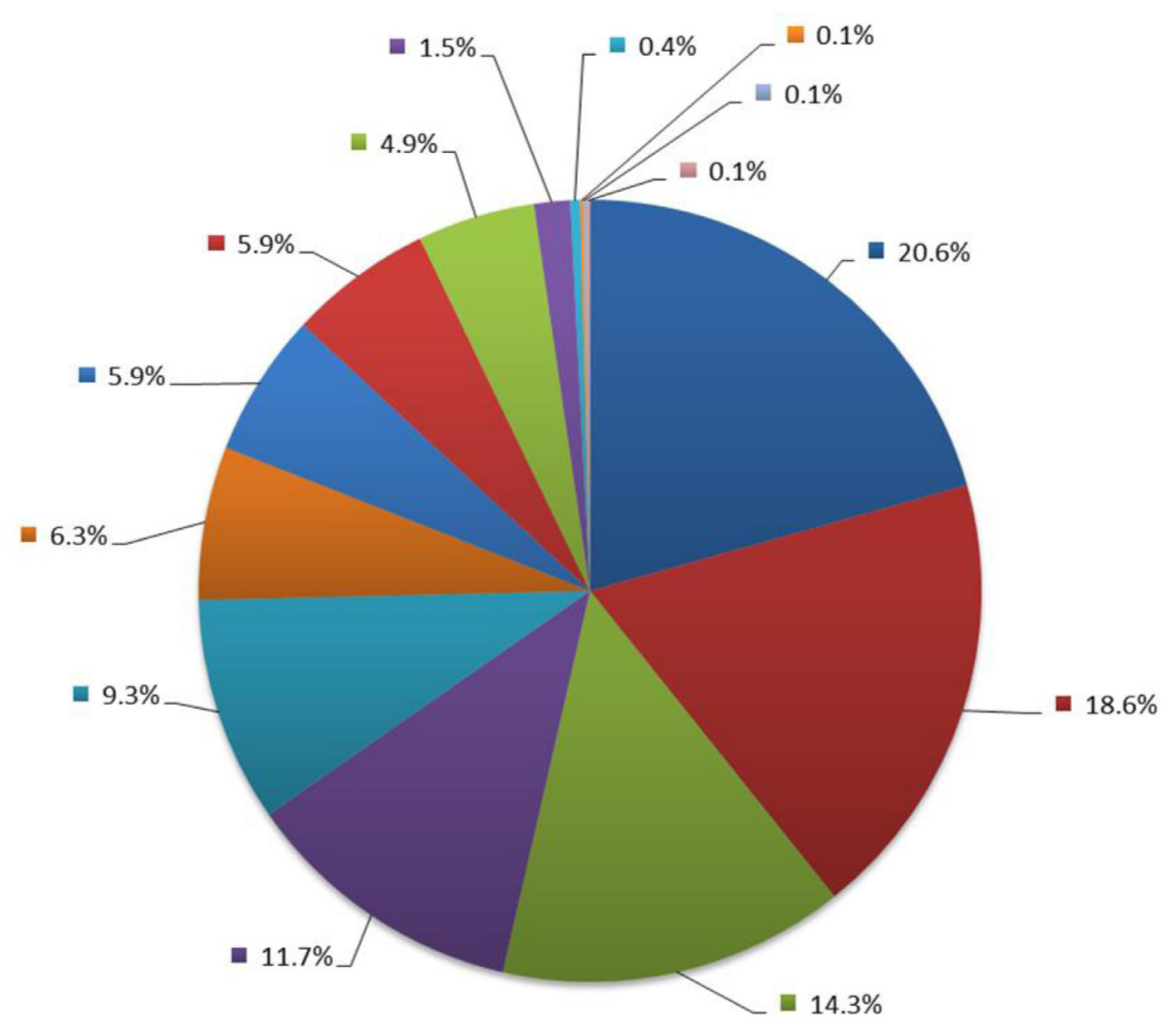

Figure 1: Pie chart of the distribution of institution contributions. 
Table 1: Summary of the clinical series and sequencing performances

\begin{tabular}{lccccc}
\hline & Primary & Metastasis & Unknown & \multicolumn{2}{c}{ Total } \\
\cline { 2 - 6 } & $\boldsymbol{N}$ & $\boldsymbol{N}$ & $\boldsymbol{N}$ & $\boldsymbol{N}$ & $\mathbf{( \% )}$ \\
\hline Primary Tumor/Metastasis & 584 & 112 & 45 & & 741 \\
Sample Types $(\boldsymbol{n}=\mathbf{7 0 8})$ & & & & & \\
$\quad$ Cell block & 0 & 7 & 0 & 7 & $(1.0)$ \\
$\quad$ Biopsy & 256 & 43 & 12 & 311 & $(43.9)$ \\
$\quad$ Surgical resection & 316 & 58 & 16 & 390 & $(55.1)$ \\
\% of Tumor Cells $(\boldsymbol{n}=\mathbf{7 3 5})$ & & & & & \\
$\quad<10 \%$ & 45 & 8 & 2 & 55 & $(7.5)$ \\
$\quad 10-50 \%$ & 463 & 62 & 34 & 559 & $(76.1)$ \\
$\quad>50 \%$ & 71 & 41 & 9 & 121 & $(16.5)$ \\
Sequencing Performance $(\boldsymbol{n}=\mathbf{7 4 1 )}$ & & & & & \\
$\quad$ Non-informative & 8 & 6 & 0 & 14 & $(1.9)$ \\
$\quad$ Informative & 576 & 106 & 45 & 727 & $(98.1)$ \\
\hline
\end{tabular}

is faster and requires less DNA than would be needed for traditional methods.

In the present study, we report a large series of CRC patients for whom the tumor was prospectively analyzed in a daily practice setting using targeted NGS. Of 741 consecutive CRC patients, the molecular profiling of the tumor was successful for $98 \%$.

Coverage analysis allowed us to suggest highlevel amplification of ERBB2 or EGFR genes, as already described [14]. However, this technology has to be further validated for copy number variation analysis in order to determine its sensitivity and specificity. In the present study, all suggested amplifications were confirmed by ISH. However, we can't be sure that all amplifications (especially low level amplification) are detected.

One of the critical steps in implementing new technology in routine testing is TAT. International guidelines recommend that RAS results should be available within 7-10 working days of receiving the specimen in the testing laboratory for $>90 \%$ of the samples [7-9]. Our median TAT is of 8 calendar days and tends to decrease with years (median TAT of 11 calendar days in 2013 and 8 calendar days in 2015). The TAT is often increased by the fact that samples need to be pooled in order to fill a run once a week. If a request arrives in the laboratory one day too late for DNA extraction, the test is postponed to the next week. A potential solution would be to perform more than one run per week. However, this clinical scenario would be possible only if the number of samples to test increased in order to fill out the run and be cost-effective.

The molecular profiling of CRC tumors reported in the present study is similar to that reported in the literature and public databases $[6,15-20]$. We are able to identify potentially actionable mutations for 464 patients, most of them carrying KRAS and/or NRAS mutations (365). According to international guidelines [2, 4], the

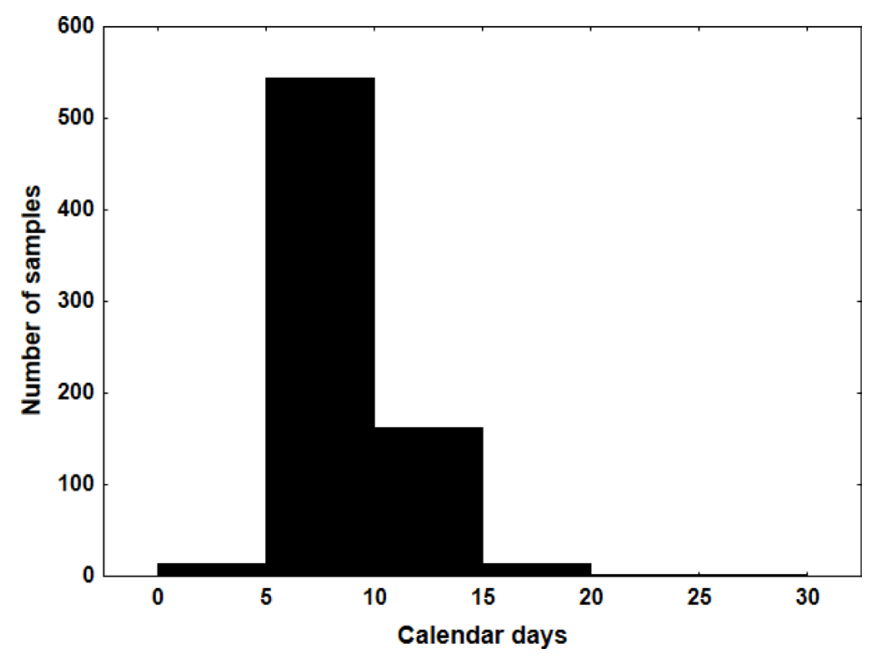

Figure 2: Histogram of the turnaround time measured in calendar days. 
Table 2: Summary of the potentially actionable mutations identified $(N=563)$ and the percentage of patients with mutations in each gene

\begin{tabular}{|c|c|c|c|c|c|}
\hline \multirow{2}{*}{ Gene } & \multicolumn{4}{|c|}{ Number of mutations } & \multirow{2}{*}{$\%$ patients with mutations } \\
\hline & PRIMARY & METASTASIS & UNKNOWN & TOTAL & \\
\hline $\mathrm{KRAS}^{*}$ & 268 & 50 & 25 & 343 & $46.1 \%(335 / 727)$ \\
\hline p.G12V/D/A/S/C/R/F & 189 & 33 & 12 & 234 & \\
\hline p.G13D/R/S/C & 41 & 6 & 7 & 54 & \\
\hline p.L19F & 0 & 0 & 1 & 1 & \\
\hline p.Q22K & 1 & 0 & 2 & 3 & \\
\hline p.A59T & 1 & 0 & 1 & 2 & \\
\hline p.Q61K/H/L & 7 & 2 & 0 & 9 & \\
\hline p.K117N & 4 & 2 & 0 & 6 & \\
\hline p.A146T/V/G/P & 25 & 7 & 2 & 34 & \\
\hline NRAS & 26 & 4 & 2 & 32 & $4.4 \%(32 / 727)$ \\
\hline p.G12V/D/S/C & 8 & 1 & 1 & 10 & \\
\hline p.G13R/D/C/V & 5 & 1 & 0 & 6 & \\
\hline p.A59T & 0 & 1 & 0 & 1 & \\
\hline p.Q61K/L/H/R & 13 & 1 & 1 & 15 & \\
\hline BRAF & 63 & 11 & 4 & 78 & $10.7 \%(78 / 727)$ \\
\hline p.R462I & 0 & 0 & 1 & 1 & \\
\hline p.G466A/E/R & 3 & 0 & 0 & 3 & \\
\hline p.G469E & 1 & 0 & 0 & 1 & \\
\hline p.D594G/N & 4 & 1 & 0 & 5 & \\
\hline p.F595L & 1 & 0 & 0 & 1 & \\
\hline p.V600E & 54 & 10 & 3 & 67 & \\
\hline PIK3CA $^{*}$ & 81 & 15 & 5 & 101 & $13.8 \%(100 / 727)$ \\
\hline p.E542K/V & 9 & 2 & 1 & 12 & \\
\hline p.E545K/G/Q & 38 & 1 & 1 & 40 & \\
\hline p.Q546P/K/L/H/R & 11 & 3 & 1 & 15 & \\
\hline p.Q564P & 1 & 0 & 0 & 1 & \\
\hline p.Y1021C & 1 & 0 & 0 & 1 & \\
\hline p.H1047R/Y & 21 & 9 & 2 & 32 & \\
\hline AKT1 & 3 & 1 & $\mathbf{0}$ & 4 & $0.6 \%(4 / 727)$ \\
\hline p.E17K & 3 & 1 & 0 & 4 & \\
\hline ERBB2 & 3 & $\mathbf{0}$ & $\mathbf{0}$ & 3 & $0.4 \%(3 / 727)$ \\
\hline p.D762Y & 1 & 0 & 0 & 1 & \\
\hline p.V777L & 1 & 0 & 0 & 1 & \\
\hline p.V842I & 1 & 0 & 0 & 1 & \\
\hline МАР2K1 & 1 & $\mathbf{0}$ & $\mathbf{0}$ & 1 & $0.1 \%(1 / 727)$ \\
\hline p.K57N & 1 & 0 & 0 & 1 & \\
\hline EGFR & $\mathbf{0}$ & 1 & $\mathbf{0}$ & 1 & $0.1 \%(1 / 727)$ \\
\hline p.D761N & 0 & 1 & 0 & 1 & \\
\hline
\end{tabular}


presence of a RAS mutation is a contraindication to antiEGFR therapy. Nevertheless, the discovery of particular mutations in rare genes can cause a change in the treatment of the patient by including him/her in a clinical trial or medical need program. According to our data, $14 \%$ of the patients (99/727) qualify for enrolment in a clinical trial. However, given the fact that the patients in the present study come from different institutions, we are not able to record the outcome of the patients or the exact proportion of patients enrolled in a clinical trial.

In conclusion, the requirements for implementation of a new test in daily practice include that (i) the test must be performed on routine samples with low DNA content, (ii) the test results must be delivered rapidly, and (iii) the test results must be accurate and facilitate clinical decision-making. The present study shows that targeted

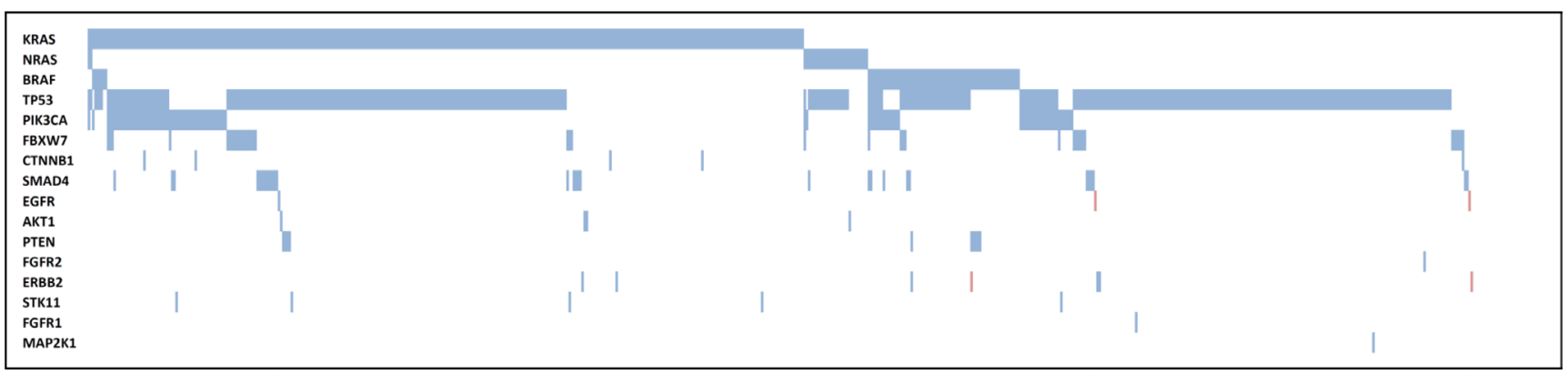

Figure 3: Molecular profile of CRC samples. Molecular alterations in different genes (rows) are indicated for each CRC sample (columns). A full square indicates that a mutation was found (in the gene), a hatched square indicates that an amplification was found (in the gene), whereas an empty square indicated that no mutation was detected (in the gene).

A

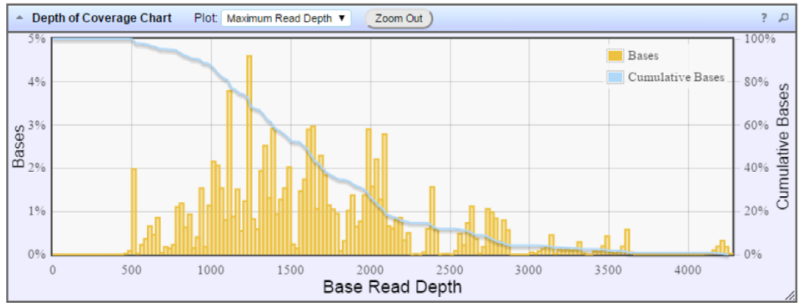

C

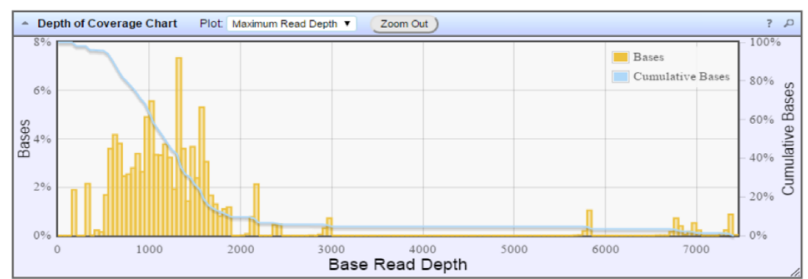

$\mathrm{E}$

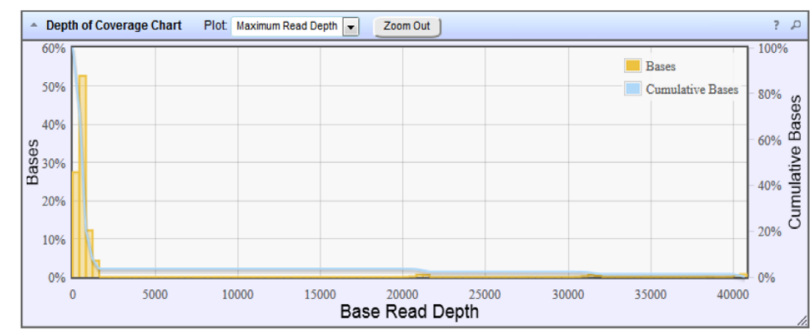

B

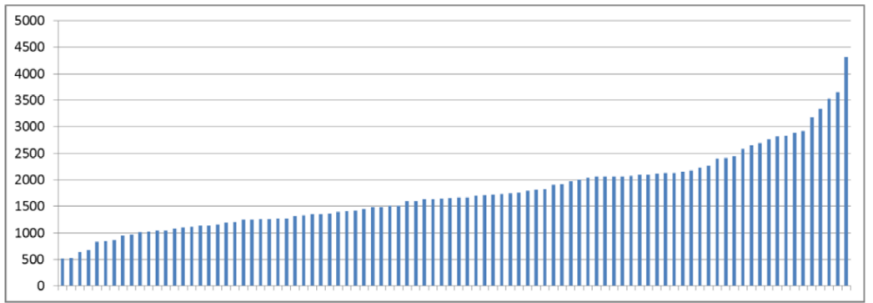

D

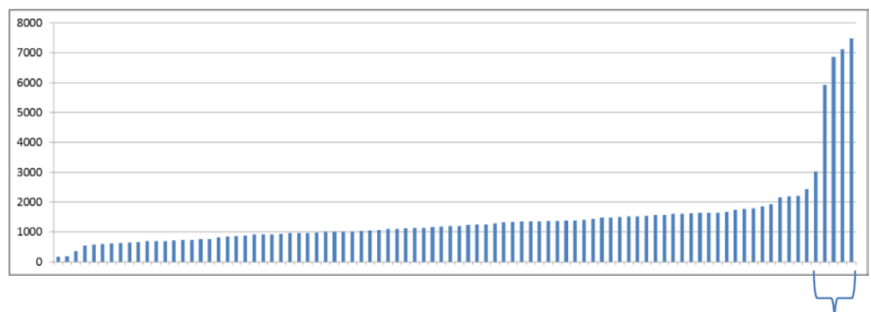

5/5 EGFR amplicons

F

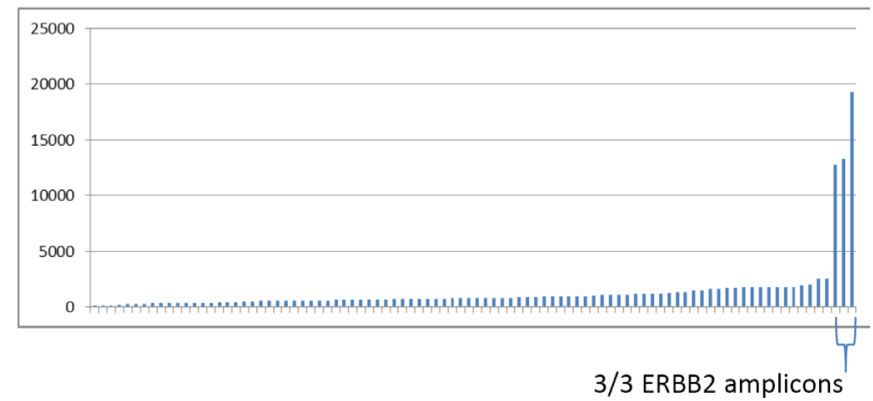

Figure 4: Histograms of the distribution of base read depth $(\mathbf{A}, \mathbf{C}, \mathbf{E})$ and of the distribution of amplicon read depth $(\mathbf{B}, \mathbf{D}, \mathbf{F})$ for a "typical" case (A, B) without gene amplification, for an EGFR-amplified case (C, D) and for an ERBB2-amplified case (E, F). 
NGS is suitable for use in clinical daily practice. This new technology is very attractive because it provides a mutational profile of a tumor, leading to precision medicine.

\section{MATERIALS AND METHODS}

\section{Samples}

Tumor samples from 741 patients from 14 different institutions (private laboratories, academic and nonacademic centers) for whom molecular testing was requested by medical oncologists were analyzed in a daily routine practice. Data were prospectively collected. DNA was extracted from FFPE tumor samples, after macrodissection of the tumor area, using the QIAamp FFPE tissue kit (Qiagen, Antwerp, Belgium) according to the manufacturer's instructions. The H\&E stained slide from the same block, previously reviewed by a pathologist who circled the tumor area and evaluated the tumor percentage, was used as a guide for the macrodissection. The DNA obtained was quantified using the Qubit ${ }^{\circledR}$ fluorometer in combination with the Qubit ${ }^{\mathbb{B}}$ dsDNA HS assay kit (Life Technologies, Gent, Belgium).

\section{Next-generation sequencing}

NGS was performed as previously described $[13,21]$. Briefly, $10 \mathrm{ng}$ of DNA was amplified using the Colon and Lung Cancer panel (Ampliseq ${ }^{\mathrm{TM}}$, Life Technologies) in order to sequence 1825 hotspot mutations in 22 genes (90 amplicons) including AKT1, ALK, BRAF, CTNNB1, DDR2, EGFR, ERBB2, ERBB4, FBXW7, FGFR1, FGFR2, FGFR3, KRAS, MAP2K1, MET, NOTCH1, NRAS, PIK3CA, PTEN, SMAD4, STK11, TP53. Sequencing was performed on a PGM $^{\mathrm{TM}}$ sequencer. The raw data were analyzed using the torrent suite software (v4.0 to v5.0 - Life Technologies). The coverage analysis was performed using the coverage analysis plug-in. Cases for which the number of mapped reads was $<100,000$ and/or the average base coverage was $<500 \times$ were considered as non-informative. Mutations were detected using the Variant Caller plug-in (v4.0 to v5.0) with low stringency settings (Life Technologies). In the variant list obtained, we considered a variant as authentic if the variant coverage was at least $30 \times$ and if the variant frequency was at least $4 \%[13,21]$. Moreover, each mutation was verified in the Integrative genome viewer (IGV) from the Broad Institute (http://www.broadinstitute. org/igv/). Only mutations reported in the COSMIC (Sanger Institute Catalogue of Somatic Mutations in Cancer) database (http://www.sanger.ac.uk/cosmic) were taken into account, and silent or intronic mutations were not reported. A case was considered to be without mutation if the tumor content was at least $10 \%$ tumor cells, if the case was considered informative, and if no mutation was detected. Moreover, hotspot regions in KRAS (exons 2, 3, 4), NRAS (exons 2, 3, 4) and BRAF (exon 15) were manually verified for each case in the Integrative Genome Viewer (IGV) from the Broad Institute (http://www.broadinstitute.org/igv/). Mutation detection sensitivity was controlled for by including either the AcroMetrix ${ }^{\mathrm{TM}}$ Oncology Hotspot Control (Life Technologies) or the Tru-Q HDx ${ }^{\mathrm{TM}}$ Reference Standard (Horizon Discovery, Cambridge, UK) in each of the Ion $\mathrm{PGM}^{\mathrm{TM}}$ runs.

The method used (including DNA extraction, sequencing and data analysis) is ISO 15189 certified.

\section{Gene amplification evaluation}

Coverage analysis of the NGS data allowed us to evaluate potential high level amplifications of ERBB2 (HER-2) and EGFR genes. Gene amplification was suggested when coverage analysis revealed a deviation in depth of coverage for all the amplicons covering ERBB2 or EGFR (Figure 4). For these cases, in situ hybridization was performed to confirm the gene amplification.

\section{In situ hybridization}

When coverage analysis suggested an amplification of ERBB2 (HER-2) or EGFR genes, in situ hybridization (ISH) was performed for confirmation. Fresh cut, 4- $\mu \mathrm{m}$ sections of FFPE tissue were submitted to dual-color ISH using probe sets. HER-2 fluorescence ISH was performed using the PathVysion HER-2 DNA Probe Kit (Abbott, Wavre, Belgium) according to the manufacturer's instructions. EGFR chromogenic ISH was performed using the ZytoDot ${ }^{\circledR}$ 2C SPEC EGFR/CEN 7 Probe (Zytovision, Bremerhaven, Germany) according to the manufacturer's instructions.

\section{Author contributions}

$\mathrm{ND}$, analysis and interpretation of data, manuscript writing; QF, data collection, manuscript writing; NDN, Acquisition of data, analysis and interpretation of data; OB, Acquisition of data, analysis and interpretation of data; BM, manuscript writing and reviewing; MD, provides CRC samples; $\mathrm{M}-\mathrm{FD}$, provides CRC samples; CM, provides CRC samples; NN, provides CRC samples; ER, provides CRC samples; JV, provides CRC samples; AG, provides CRC samples; CDP, provides CRC samples; LV, provides CRC samples; M-PVC, provides CRC samples; $\mathrm{PD}$, provides CRC samples; J-LVL, manuscript reviewing; IS, study supervision and manuscript reviewing; MLM, analysis and interpretation of data, manuscript reviewing. 


\section{CONFLICTS OF INTEREST}

The authors have declared no conflicts of interest.

\section{FUNDING}

This work was supported by funding from the "Fonds Yvonne Boël” (Brussels, Belgium).

\section{REFERENCES}

1. Ferlay J, Steliarova-Foucher E, Lortet-Tieulent J, Rosso S, Coebergh JW, Comber H, Forman D, Bray F. Cancer incidence and mortality patterns in Europe: estimates for 40 countries in 2012. Eur J Cancer. 2013; 49:1374-1403.

2. Van Cutsem E, Cervantes A, Nordlinger B, Arnold D. Metastatic colorectal cancer: ESMO Clinical Practice Guidelines for diagnosis, treatment and follow-up. Ann Oncol. 2014; 25:1-9.

3. Shi C, Washington K. Molecular testing in colorectal cancer: diagnosis of Lynch syndrome and personalized cancer medicine. Am J Clin Pathol. 2012; 137:847-859.

4. Allegra CJ, Rumble RB, Hamilton SR, Mangu PB, Roach N, Hantel A, Schilsky RL. Extended RAS Gene Mutation Testing in Metastatic Colorectal Carcinoma to Predict Response to Anti-Epidermal Growth Factor Receptor Monoclonal Antibody Therapy/ American Society of Clinical Oncology Provisional Clinical Opinion Update 2015. J Clin Oncol. 2016; 34:179-185.

5. Pillai RK, Lopategui JR, Dhall D, Guindi M, Slavin T, Lofton-Day CE, Patterson SD. The State of the Art in Colorectal Cancer Molecular Biomarker Testing. Adv Anat Pathol. 2016; 23:92-103.

6. Wong NA, Gonzalez D, Salto-Tellez M, Butler R, DiazCano SJ, Ilyas M, Newman W, Shaw E, Taniere P, Walsh SV, and Association of Clinical Pathologists Molecular Pathology and Diagnostics Group. RAS testing of colorectal carcinoma - a guidance document from the Association of Clinical Pathologists Molecular Pathology and Diagnostics Group. J Clin Pathol. 2014; 67:751-757.

7. Jouret-Mourin A, Cuvelier C, Demetter P, D'Haene N, Driessen N, Hoorens A, Nagy N, Sagaert X, Pauwels P. RAS-testing in colorectal cancer: Belgian guidelines. Belg J Med Oncol. 2015; 9:183-190.

8. Van Krieken JH, Rouleau E, Ligtenberg MJ, Normanno N, Patterson SD, Jung A. RAS testing in metastatic colorectal cancer: advances in Europe. Virchows Arch. 2016; 468:383-396.

9. van Krieken JH, Siebers AG, Normanno N. European consensus conference for external quality assessment in molecular pathology. Ann Oncol. 2013; 24:1958-1963.

10. McCourt CM, McArt DG, Mills K, Catherwood MA, Maxwell P, Waugh DJ, Hamilton P, O'Sullivan JM, Salto-Tellez M. Validation of next generation sequencing technologies in comparison to current diagnostic gold standards for BRAF, EGFR and KRAS mutational analysis. PLoS One. 2013; 8:e69604.
11. Tuononen K, Maki-Nevala S, Sarhadi VK, Wirtanen A, Ronty M, Salmenkivi K, Andrews JM, Telaranta-Keerie AI, Hannula S, Lagstrom S, Ellonen P, Knuuttila A, Knuutila S. Comparison of targeted next-generation sequencing (NGS) and real-time PCR in the detection of EGFR, KRAS, and BRAF mutations on formalin-fixed, paraffin-embedded tumor material of non-small cell lung carcinoma-superiority of NGS. Genes Chromosomes Cancer. 2013; 52:503-511.

12. Scarpa A, Sikora K, Fassan M, Rachiglio AM, Cappellesso R, Antonello D, Amato E, Mafficini A, Lambiase M, Esposito C, Bria E, Simonato F, Scardoni M, et al. Molecular typing of lung adenocarcinoma on cytological samples using a multigene next generation sequencing panel. PLoS One. 2013; 8:e80478.

13. D'Haene N, Le Mercier M, De Neve N, Blanchard O, Delaunoy M, El Housni H, Dessars B, Heimann P, Remmelink M, Demetter P, Tejpar S, Salmon I. Clinical Validation of Targeted Next Generation Sequencing for Colon and Lung Cancers. PLoS One. 2015; 10:e138245.

14. Hoogstraat M, Hinrichs JW, Besselink NJ, Radersma-van Loon JH, de Voijs CM, Peeters T, Nijman IJ, de Weger RA, Voest EE, Willems SM, Cuppen E, Koudijs MJ. Simultaneous detection of clinically relevant mutations and amplifications for routine cancer pathology. J Mol Diagn. 2015; 17:10-18.

15. Dienstmann R, Serpico D, Rodon J, Saura C, Macarulla T, Elez E, Alsina M, Capdevila J, Perez-Garcia J, Sanchez-Olle G, Aura C, Prudkin L, Landolfi S, et al. Molecular profiling of patients with colorectal cancer and matched targeted therapy in phase I clinical trials. Mol Cancer Ther. 2012; 11:2062-2071.

16. El-Deiry WS, Vijayvergia N, Xiu J, Scicchitano A, Lim B, Yee NS, Harvey HA, Gatalica Z, Reddy S. Molecular profiling of 6,892 colorectal cancer samples suggests different possible treatment options specific to metastatic sites. Cancer Biol Ther. 2015; 16:1726-1737.

17. Jesinghaus M, Pfarr N, Endris V, Kloor M, Volckmar AL, Brandt R, Herpel E, Muckenhuber A, Lasitschka F, Schirmacher P, Penzel R, Weichert W, Stenzinger A. Genotyping of colorectal cancer for cancer precision medicine: Results from the IPH Center for Molecular Pathology. Genes Chromosomes Cancer. 2016; 55:505-521.

18. Malapelle U, Pisapia P, Sgariglia R, Vigliar E, Biglietto M, Carlomagno C, Giuffre G, Bellevicine C, Troncone G. Less frequently mutated genes in colorectal cancer: evidences from next-generation sequencing of 653 routine cases. J Clin Pathol. 2016; 69:767-771.

19. Malapelle U, Vigliar E, Sgariglia R, Bellevicine C, Colarossi L, Vitale D, Pallante P, Troncone G. Ion Torrent next-generation sequencing for routine identification of clinically relevant mutations in colorectal cancer patients. J Clin Pathol. 2014; 68:64-68.

20. Cancer Genome Atlas Network. Comprehensive molecular characterization of human colon and rectal cancer. Nature. 2012; 487:330-337.

21. Le Mercier M, D'Haene N, De Neve N, Blanchard O, Degand C, Rorive S, Salmon I. Next-generation sequencing improves the diagnosis of thyroid FNA specimens with indeterminate cytology. Histopathology. 2015; 66:215-224. 